\title{
Magnetic resonance imaging of ultrasmall superparamagnetic iron oxide-labeled exosomes from stem cells: a new method to obtain labeled
} exosomes

\author{
This article was published in the following Dove Press journal: \\ International Journal of Nanomedicine \\ I June 2016 \\ Number of times this article has been viewed
}

\author{
Alice Busato',* \\ Roberta Bonafedel,* \\ Pietro Bontempi ${ }^{2}$ \\ Ilaria Scambi' \\ Lorenzo Schiaffino' \\ Donatella Benati' \\ Manuela Malatesta' \\ Andrea Sbarbati' \\ Pasquina Marzola ${ }^{3}$ \\ Raffaella Mariotti' \\ 'Department of Neurosciences, \\ Biomedicine and Movement Sciences, \\ School of Medicine, ${ }^{2}$ Department \\ of Biotechnology, ${ }^{3}$ Department of \\ Computer Science, University of \\ Verona, Verona, Italy \\ *These authors contributed equally \\ to this work
}

Purpose: Recent findings indicate that the beneficial effects of adipose stem cells (ASCs), reported in several neurodegenerative experimental models, could be due to their paracrine activity mediated by the release of exosomes. The aim of this study was the development and validation of an innovative exosome-labeling protocol that allows to visualize them with magnetic resonance imaging (MRI).

Materials and methods: At first, ASCs were labeled using ultrasmall superparamagnetic iron oxide nanoparticles (USPIO, 4-6 nm), and optimal parameters to label ASCs in terms of cell viability, labeling efficiency, iron content, and magnetic resonance (MR) image contrast were investigated. Exosomes were then isolated from labeled ASCs using a standard isolation protocol. The efficiency of exosome labeling was assessed by acquiring MR images in vitro and in vivo as well as by determining their iron content. Transmission electron microscopy images and histological analysis were performed to validate the results obtained.

Results: By using optimized experimental parameters for ASC labeling $(200 \mu \mathrm{g} \mathrm{Fe} / \mathrm{mL}$ of USPIO and 72 hours of incubation), it was possible to label $100 \%$ of the cells, while their viability remained comparable to unlabeled cells; the detection limit of MR images was of $10^{2}$ and $2.5 \times 10^{3} \mathrm{ASCs}$ in vitro and in vivo, respectively. Exosomes isolated from previously labeled ASCs retain nanoparticles, as demonstrated by transmission electron microscopy images. The detection limit by MRI was $3 \mu \mathrm{g}$ and $5 \mu \mathrm{g}$ of exosomes in vitro and in vivo, respectively.

Conclusion: We report a new approach for labeling of exosomes by USPIO that allows detection by MRI while preserving their morphology and physiological characteristics.

Keywords: MRI, superparamagnetic iron oxide nanoparticles, cellular imaging, stem cells labeling, exosome labeling

\section{Introduction}

Mesenchymal stem cells are adult multipotent stem cells capable of self-renewal and differentiation. Among the different mesenchymal stem cells that can be isolated, adipose stem cells (ASCs) are of great interest for potential therapeutic applications due to easy availability, to their ability to migrate to damaged tissue, to their capability to differentiate, and contribution in reparative processes. ${ }^{1-3}$ Moreover, it has been recently demonstrated that in vivo administration of ASCs ameliorates the disease condition in several neurodegenerative animal models. ${ }^{4-6}$ In particular, it has been reported that transplantation of autologous ASCs has a therapeutic effect in experimental autoimmune encephalomyelitis and in murine model of familial amyotrophic 
lateral sclerosis. ${ }^{5,6}$ Literature findings indicate that ASCs exert their action through paracrine activity rather than through engraftment. ${ }^{7-9}$ Furthermore, this paracrine activity is mediated by the release of membrane vesicles, such as exosomes. ${ }^{10}$ These vesicles are from $30 \mathrm{~nm}$ to $100 \mathrm{~nm}$ in size and are of endosomal origin; ${ }^{11}$ for this reason, they contain typical endosome-associated proteins, such as tetraspanin, alix, and heat-shock proteins, some of which are used as specific and quantitative endosomal markers. ${ }^{12}$ Exosomes contain lipids, proteins, and nucleic acid (in particular, mRNA, tRNA, miRNA, and noncoding RNA) that can be transferred to cells and regulate their activities. ${ }^{12,13}$ Based on the accumulating evidence for the key role of exosomes in neuronal protection, nerve regeneration, remyelination, and synaptic plasticity, ${ }^{14-17}$ several authors support the idea that exosomes can recapitulate the neuroprotective effect of stem cells and suggest their use for a noncell-based therapy of neurodegenerative diseases. ${ }^{3,15-17}$ Nevertheless, elucidation of the action mechanism of both ASCs and exosomes requires knowledge of their homing in the recipient body.

In order to understand where stem cells or exosomes exert their therapeutic effect, the ability to track cells and exosomes in a noninvasive manner, with an appropriate imaging method, such as magnetic resonance imaging (MRI), becomes crucial. Recently, nanotechnologies have contributed to advances in in vivo high-resolution imaging to monitor stem cells and exosomes tracking and homing, providing useful insights in their mechanism of action. ${ }^{18-23}$ To produce a detectable change in signal intensity, cells or exosomes must be labeled with magnetic resonance (MR) contrast agents. In this regard, superparamagnetic iron oxide nanoparticles, which are small crystalline magnetite structures ranging in size from $5 \mathrm{~nm}$ to $150 \mathrm{~nm}$, have been widely used to magnetically label stem cells and exosomes. ${ }^{23,24}$

To the best of our knowledge, a single method to label exosomes for MRI has been reported: exosomes are loaded with nanoparticles through electroporation. ${ }^{23,25}$ Electroporation is an old method that has been used for many years. It uses a long electric pulse to temporarily disturb the phospholipid bilayer, creating temporary pores in membranes, allowing molecules, such as nanoparticles, to pass into the exosomes. A temporary breakage of the membrane may alter their protein composition that might affect the function of the exosomes. Therefore, it is essential to find an alternative method that preserves membrane integrity.

Here, we set up an innovative approach for exosome labeling that preserves their morphology and physiological characteristics. Specifically, we show that by labeling ASCs with ultrasmall superparamagnetic iron oxide nanoparticles
(USPIO, 4-6 nm) before vesicles extraction, the isolated exosomes retain nanoparticles and can be visualized by MRI. This study aims at validating this new USPIO-based exosome labeling method by monitoring the efficiency of the labeling with MRI both in ASCs and in extracted exosomes. We also demonstrate the value of the MRI-based approach, which allowed us to measure small amounts of USPIO-labeled cells or exosomes in in vitro and in vivo conditions.

\section{Materials and methods Cell culture}

Murine ASCs were isolated from inguinal adipose tissues of male C57BL/ 6 mice (Charles River Laboratories, Wilmington, MA, USA), as previously described. ${ }^{2}$ Briefly, the lipoaspirate was incubated in Hank's Balanced Salt Solution (Thermo Fisher Scientific, Waltham, MA, USA) with collagenase type I (Thermo Fisher Scientific) and bovine serum albumin (PanReac AppliChem, Darmstadt, Germany). After incubation, a stromal vascular fraction was obtained by ultracentrifugation, resuspended in $\mathrm{NH}_{4} \mathrm{Cl}$, centrifuged, and filtered to remove cell debris. Cells were cultured using Dulbecco's Modified Eagle's Medium with $10 \%$ fetal bovine serum (FBS), $100 \mathrm{U} / \mathrm{mL}$ penicillin, and $100 \mu \mathrm{g} / \mathrm{mL}$ streptomycin (all from Thermo Fisher Scientific) and incubated at $37^{\circ} \mathrm{C} / 5 \% \mathrm{CO}_{2}$.

\section{Iron oxide nanoparticles labeling of ASCs}

Commercial USPIO (magnetite $\mathrm{Fe}_{3} \mathrm{O}_{4}$; Sigma-Aldrich Co., St Louis, MO, USA; catalog \#725331, particle size 4-6 nm, stock solution $5 \mathrm{mg} \mathrm{Fe} / \mathrm{mL}$ ) were used to label ASCs (ASC-USPIO). The experiment was designed with two approaches. In the first approach, $10^{5}$ cells were incubated with increasing concentrations of nanoparticles (equivalent to $0,12.5,25,50,100,200 \mu \mathrm{g} \mathrm{Fe} / \mathrm{mL}$ ) at different time points (24 hours and 72 hours). USPIO were diluted in culture medium (Dulbecco's Modified Eagle's Medium with 10\% FBS, $100 \mathrm{U} / \mathrm{mL}$ penicillin, and $100 \mu \mathrm{g} / \mathrm{mL}$ streptomycin). After incubation, cells were washed twice with phosphatebuffered saline (PBS), trypsinized, and counted, and $10^{5}$ cells were resuspended in a gel matrix. In the second approach, $200 \mu \mathrm{g} \mathrm{Fe} / \mathrm{mL}$ of USPIO were used to label a variable cell concentration (from $10^{2}$ cells to $10^{5}$ cells) for 72 hours. After incubation, cells were washed twice with PBS, trypsinized, counted, and resuspended at the appropriate cell number (from $10^{2}$ cells to $10^{5}$ cells).

Cytotoxicity of nanoparticles was determined by Trypan blue (TB) exclusion assay: cells were washed twice with PBS, trypsinized, and incubated with TB dye solution. The TB dye stains dead cells in blue color, while the live cells remain unstained. Cell viability was analyzed with univariate 
analysis of variance; $P<0.05$ was considered as statistically significant.

\section{Efficiency of ASC labeling}

To verify the internalization of nanoparticles by ASCs, cells were stained with Prussian blue (PB; Perls' acid ferrocyanide) method and counterstained with nuclear fast red. Cells were fixed with a $2 \%$ solution of glutaraldehyde/paraformaldehyde for 30 minutes at room temperature and washed twice with PBS. The cells were incubated for 40 minutes in a solution of $1 \% \mathrm{HCl}$ and $2 \%$ potassium ferrocyanide, washed twice with distilled water, and counterstained using nuclear fast red for 15 minutes. Cells were then washed twice with distilled water and finally embedded in a mounting medium (Dako Mounting Medium; Agilent Technologies, Santa Clara, CA, USA). The nanoparticles internalization was investigated using a light microscope (Olympus BXS1): iron nanoparticles appeared as blue spots inside the cells, while the nucleus appeared red. The cells that exhibit red nucleus and blue intracellular particles were considered PB positive; the percentage of labeled cells was calculated within eight random fields of view per slide. The results were analyzed with univariate analysis of variance corrected for multiple comparisons (Bonferroni). $P<0.05$ was considered as statistically significant.

\section{Isolation and characterization of labeled exosomes}

ASCs ( $10^{6}$ cells) were incubated with $200 \mu \mathrm{g} \mathrm{Fe} / \mathrm{mL}$ of USPIO for 24 hours, washed, and deprived of FBS for 48 hours to avoid any contamination of vesicles from serum. After deprivation, ASC supernatants were collected, and exosomes were isolated using PureExo ${ }^{\circledR}$ Exosome isolation kit (101Bio, Palo Alto, CA, USA). The concentration of exosomes was measured in terms of their protein content determined by bicinchoninic protein assay method using the manufacturer's protocol (Pierce BCA Protein Assay Kit; Thermo Fisher Scientific). Exosomes loaded with USPIO (exosomes-USPIO) were characterized by electron microscopy (as described in "Transmission electron microscopy" section) and Western blot analysis. Western blot hybridization was performed using standard protocols: after denaturation, $12 \mu \mathrm{g}$ of proteins were separated on $4 \%-12 \%$ polyacrylamide gels, transferred on to a nitrocellulose membrane, and incubated with antibodies anti-HSP70 and antiAlix (HSP70 [K-20]: sc-1060 and Alix [Q-19]: sc-49268) (all antibodies were purchased from Santa Cruz Biotechnology Inc., Dallas, TX, USA). ASC lysate was used as a positive control, and supernatant lacking ASCs and exosomes was used as a negative control. After incubation with appropriate horseradish peroxidase-coupled secondary antibodies (Santa Cruz Biotechnology Inc.), the blot was incubated with a chemiluminescent horseradish peroxidase substrate and detected with G:BOX F3 GeneSys (Syngene, Cambridge, UK).

\section{Transmission electron microscopy}

For ultrastructural morphology of cells and exosomes, cell pellets were fixed in 2\% glutaraldehyde in Sorensen buffer ( $\mathrm{pH} 7.4$ ) for 2 hours, while exosomes included in an agarose gel were fixed with $2.5 \%$ glutaraldehyde plus $2 \%$ paraformaldehyde in $0.1 \mathrm{M}$ phosphate buffer. All samples were postfixed in $1 \%$ osmium tetroxide $\left(\mathrm{OsO}_{4}\right)$ for 2 hours and cut. The sections were then dehydrated in graded concentrations of acetone and embedded in Epon-Araldite mixture (Electron Microscopy Sciences, Hatfield, PA, USA). The semithin sections ( $1 \mu \mathrm{m}$ in thickness) were examined by light microscopy (Olympus BX51; Olympus Corporation, Tokyo, Japan) and stained with toluidine blue. The ultrathin sections were cut at a $70 \mathrm{~nm}$ thickness and placed on $\mathrm{Cu} / \mathrm{Rh}$ grids with Ultracut E (Reichert, Wien, Austria). Transmission electron microscopy (TEM) images were acquired with a Philips Morgagni TEM operating at $80 \mathrm{kV}$ and equipped with a Megaview II camera for digital image acquisition.

\section{Iron quantification in ASCs and exosomes}

Quantification of intracellular iron nanoparticles in ASCs was performed as previously described by Rad et al. ${ }^{26}$ The cells were washed three times with PBS, trypsinized, counted, and collected in tubes $\left(2 \times 10^{5}\right.$ cells each). Cells were centrifuged at 3,000 rpm for 5 minutes and incubated with $0.5 \mathrm{~mL}$ of $\mathrm{HCl}$ $(5 \mathrm{M})$ at room temperature for 1 hour. ASCs were then centrifuged at 3,000 rpm for 5 minutes, and the supernatant was collected in $1.5 \mathrm{~mL}$ microcuvettes. Potassium ferrocyanide solution (5\%) was added to each microcuvette and incubated at room temperature for 30 minutes in dark conditions. The absorbance was measured at $700 \mathrm{~nm}$ using the ultraviolet-visible spectrophotometer (Cary $60 \mathrm{UV} / \mathrm{V}$ is Spectrophotometer; Agilent Technologies). The same protocol was performed for unlabeled cells, used as control. In order to determine the iron concentration per cell, the absorbance of each sample was divided by the number of cells per milliliter. Iron concentration was determined by normalizing the obtained absorbance with a previously prepared calibration curve. The calibration curve was obtained by preparing samples with different iron concentrations $(0-12 \mu \mathrm{g} \mathrm{Fe} / \mathrm{mL}) ; \mathrm{HCl}(5 \mathrm{M})$ was added to each sample and incubated at room temperature for 1 hour. After incubation time, potassium ferrocyanide (5\%) was added, and the tubes were kept for 30 minutes in dark conditions. The absorbance was measured at $700 \mathrm{~nm}$, and the iron 
calibration curve was derived. The same protocol used for the calibration curve was performed for the quantification of iron content in exosomes after their isolation.

\section{In vitro and in vivo $M R I$}

MR images were acquired using a Bruker Tomograph (Bruker Optik GmbH, Ettlingen, Germany) equipped with a $4.7 \mathrm{~T}$, $33 \mathrm{~cm}$ bore horizontal magnet (Oxford Ltd, Oxford, UK).

For in vitro $\mathrm{T}_{2}$ relaxation time evaluation, ASCs were labeled as previously described and then homogeneously distributed in a gel matrix (Agarose low electroendosmosis; PanReac AppliChem; at 2\% w/w). $\mathrm{T}_{2}$ maps of ASC-USPIO phantoms were obtained with a multislice multiecho sequence with repetition time $(\mathrm{TR})=5,000 \mathrm{~ms}$, echo time $\left(\mathrm{TE}_{\mathrm{eff}}\right)=11 \mathrm{~ms}, \mathrm{~N}$ echoes $=16$, field of view $($ FOV $)=6.5 \times 6.5 \mathrm{~cm}^{2}$, matrix size $($ MTX $)=128 / 128$, slice thickness $=2 \mathrm{~mm}$, and $\mathrm{N}$ slice $=3$. Relaxivity $(1 / \mathrm{s})$ of ASCUSPIO nanoparticles (R2) were analyzed by using Student's $t$-test corrected for multiple comparisons (Bonferroni). $P<0.05$ was considered as statistically significant.

In order to assess the in vitro detectability of USPIOlabeled ASCs and exosomes immobilized in a gel matrix, $\mathrm{T}_{2}{ }^{*}$-weighted images were acquired using a fast low-angle shot sequence with $\mathrm{TR}=625 \mathrm{~ms}, \mathrm{TE}_{\text {eff }}=15 \mathrm{~ms}, \mathrm{~N}$ echoes $=1$, $\mathrm{FOV}=6.0 \times 6.0 \mathrm{~cm}^{2}, \mathrm{MTX}=700 / 512$, slice thickness $=0.5 \mathrm{~mm}$, and $\mathrm{N}$ slice $=24$. Unlabeled cells or unlabeled exosomes in an agarose gel were used as the respective control.

For in vivo MRI, C57BL/6 male mice (Harlan Laboratories, Indianapolis, IN, USA) were used. Mice were maintained under standard environmental conditions (temperature, humidity, 12 hours/12 hours light/dark cycle, with water and food ad libitum) and veterinarian assistance. Animal handling was performed following a protocol approved by the Animal Care and Use Committee of the University of Verona (CIRSAL) and by the Italian Ministry of Health, in strict adherence to the European Communities Council (86/609/EEC) directives, minimizing the number of animals used and avoiding their suffering. Animals were anesthetized by $1 \%$ isofluorane inhalation in a mixture of oxygen and nitrogen and were placed in a prone position over a heated bed and inserted into a $3.5-\mathrm{cm}$ internal diameter birdcage radiofrequency coil.
In order to assess MRI detectability of labeled cells, $2.5 \times 10^{3}$ and $5 \times 10^{3}$ ASC-USPIO, suspended in $100 \mu \mathrm{L}$ of sterile PBS, were administered to two mice by intramuscular injection in the hind limbs. At the same time, in order to detect exosomes-USPIO, $5 \mu \mathrm{g}$ and $25 \mu \mathrm{g}$ of labeled exosomes (containing $0.032 \mu \mathrm{g}$ and $0.16 \mu \mathrm{g}$ Fe, respectively, and suspended in $100 \mu \mathrm{L}$ of sterile PBS) were injected intramuscularly in two additional mice. In order to confirm the power of MRI in detecting low amounts of iron, free USPIO, containing the equivalent iron content of labeled exosomes, was injected intramuscularly in two other mice. To perform in vivo MRI, $\mathrm{T}_{2}{ }^{*}$-weighted images were acquired using a fast low-angle shot sequence with $\mathrm{TR}=625 \mathrm{~ms}$, $\mathrm{TE}_{\text {eff }}=5 \mathrm{~ms}, \mathrm{FOV}=7.0 \times 3.50 \mathrm{~cm}^{2}, \mathrm{MTX}=512 / 256$, slice thickness $=0.5 \mathrm{~mm}$, and $\mathrm{N}$ slice $=24$.

\section{Histological analysis of exosomes-USPIO}

After in vivo injection, the presence of exosomes in the muscle tissues was confirmed by histological analysis. Mice were sacrificed, and the gastrocnemius was dissected out, fixed in $10 \%$ formalin, and embedded in paraffin. All samples were cut in $5 \mu \mathrm{m}$ thick sections with a microtome. In order to evaluate the nanoparticles incorporation, PB staining was performed: sections were incubated with PB solution (5\% hydrochloric acid and 5\% potassium ferrocyanide) for 40 minutes and counterstained with nuclear fast red for 10 minutes. Sections were examined under a light microscope (Olympus BXS1) equipped with a charge-coupled device camera.

\section{Results \\ Cell growth and viability}

In order to verify the possible cytotoxicity of USPIO, TB exclusion assay was performed. After ASC labeling with increasing amount of nanoparticles, no statistical differences were found, indicating that different concentrations of USPIO do not interfere with the viability of the cells (Table 1).

\section{Efficiency and iron quantification of ASC labeling}

In order to verify whether ASCs were labeled with USPIO and to detect iron nanoparticles inside the cells, PB staining

Table I Viability of ASCs (assessed by Trypan blue viability test) incubated for 24 hours and 72 hours with increasing concentrations of USPIO

\begin{tabular}{|c|c|c|c|c|c|c|}
\hline & $12.5 \mu \mathrm{g} \mathrm{Fe} / \mathrm{mL}$ & $25 \mu \mathrm{g} \mathrm{Fe} / \mathrm{mL}$ & $50 \mu \mathrm{g} \mathrm{Fe} / \mathrm{mL}$ & $100 \mu \mathrm{g} \mathrm{Fe} / \mathrm{mL}$ & $200 \mu \mathrm{g} \mathrm{Fe} / \mathrm{mL}$ & Nonlabeled \\
\hline 24 hours & $100.0 \pm 0$ & $90.7 \pm 6.3$ & $89.8 \pm 5.2$ & $93.5 \pm 4.3$ & $97.0 \pm 1.5$ & $100.0 \pm 0$ \\
\hline 72 hours & $96.2 \pm 2.1$ & $98.0 \pm 1.1$ & $97.0 \pm 2.1$ & $100.0 \pm 0$ & $92.9 \pm 2.3$ & $98.7 \pm 1.1$ \\
\hline
\end{tabular}

Note: Data are shown as mean percentage \pm SEM.

Abbreviations: ASCs, adipose stem cells; SEM, standard error of the mean; USPIO, ultrasmall superparamagnetic iron oxide nanoparticles. 
was performed. This staining is used to identify the presence of nanoparticles: the reduction of ferric to ferrous iron results in the formation of blue precipitates. PB and nuclear fast red staining of labeled cells clearly showed red nucleus and blue cytoplasmic inclusions with typical perinuclear localization indicating the presence of USPIO (Figure 1A). Labeled cells were detected after incubation using the lowest dose of nanoparticles $(12.5 \mu \mathrm{g} \mathrm{Fe} / \mathrm{mL})$ and the shortest incubation time ( 24 hours). The increase in iron concentration $(100 \mu \mathrm{g} \mathrm{Fe} / \mathrm{mL}$ and $200 \mu \mathrm{g} \mathrm{Fe} / \mathrm{mL})$ and incubation time (72 hours) resulted in a stronger PB cell staining, due to increasing amounts of USPIO internalization (Figure 1A). When ASCs were incubated 24 hours with increasing iron concentrations $(12.5 \mu \mathrm{g} \mathrm{Fe} / \mathrm{mL}, 25 \mu \mathrm{g} \mathrm{Fe} / \mathrm{mL}, 50 \mu \mathrm{g} \mathrm{Fe} / \mathrm{mL}$, $100 \mu \mathrm{g} \mathrm{Fe} / \mathrm{mL}$, and $200 \mu \mathrm{g} \mathrm{Fe} / \mathrm{mL}$ ), a consistent increase in the percentage of labeled cells was detected $(35.4 \%, 49.5 \%$, $77.6 \%, 85.8 \%$, and $87.2 \%$, respectively, at the concentrations mentioned earlier), showing a dose-dependent nanoparticle internalization (Figure 1B). After 72 hours of ASC incubation with the nanoparticle concentrations mentioned earlier, we obtained $80.2 \%, 88.2 \%, 95.8 \%, 97 \%$, and $100 \%$ of labeled cells, respectively (Figure 1B). Labeling of ASCs was found to be dose dependent and time dependent: the percentage of nanoparticles internalization increased both with increasing USPIO concentrations and with increasing incubation times.

In addition, in order to confirm the intracellular uptake of nanoparticles and visualize their intracellular localization, TEM images of ASC-USPIO were acquired (Figure 1C and D). TEM analysis showed that iron nanoparticles were accumulated inside the cells and localized exclusively in the cytoplasm. Moreover, TEM analysis revealed that USPIO were contained inside the labeled cells in endocytic vesicles (Figure 1D). TEM image of unlabeled cells as negative control was reported (Figure 1E). Iron content measures in ASC-USPIO showed intracellular concentrations of $6.20 \pm 0.0036 \mathrm{pg} / \mathrm{cell}, 7.58 \pm 0.0050 \mathrm{pg} / \mathrm{cell}$, and $9.39 \pm 0.007 \mathrm{pg} /$ cell (data expressed as mean $\pm \mathrm{SD}$ ) at concentrations of $50 \mu \mathrm{g} \mathrm{Fe} / \mathrm{mL}, 100 \mu \mathrm{g} \mathrm{Fe} / \mathrm{mL}$, and $200 \mu \mathrm{g} \mathrm{Fe} / \mathrm{mL}$, respectively, after 24 hours of incubation.
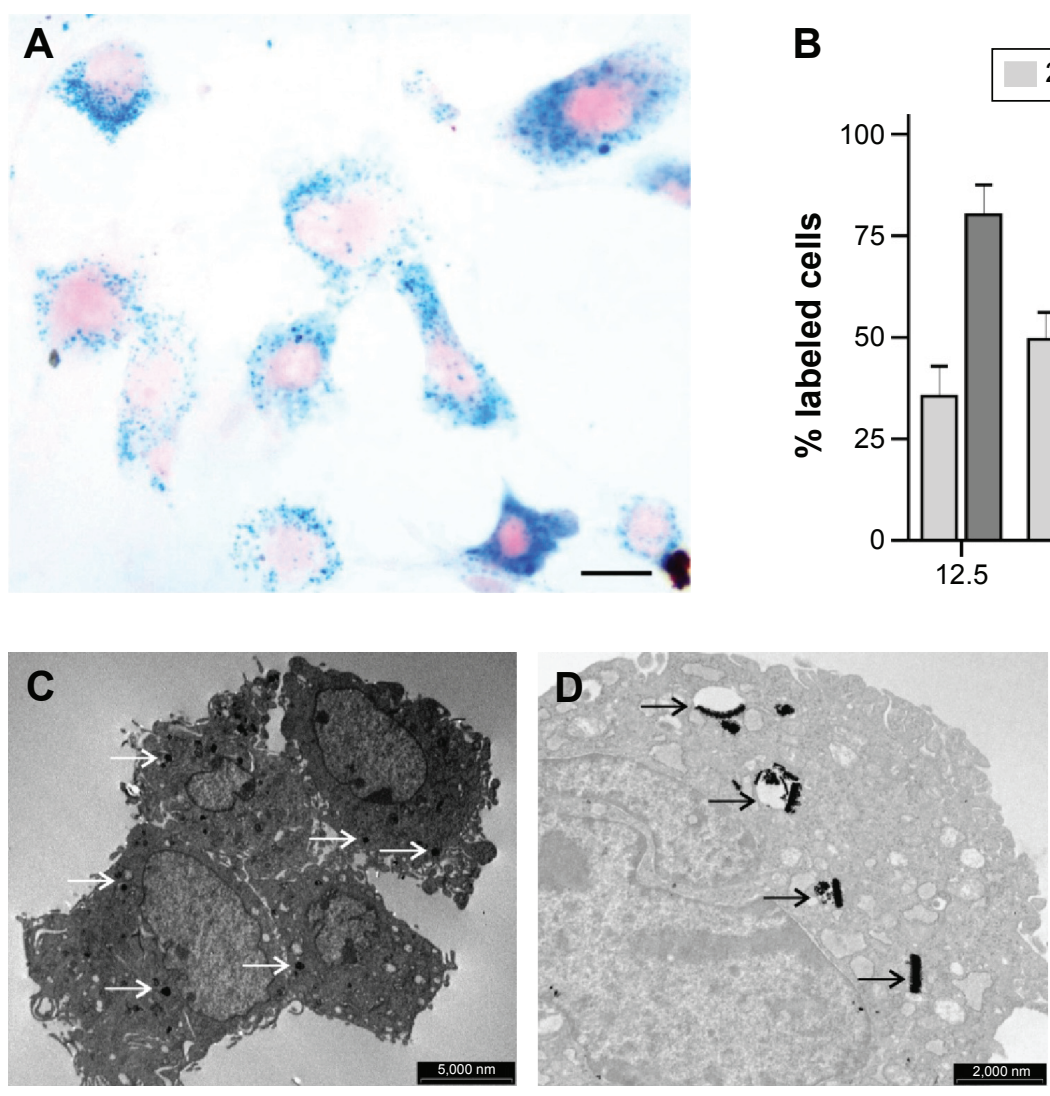

Figure I Intracellular uptake of USPIO.

Notes: (A) Prussian blue staining of ASCs to detect the presence of iron nanoparticles after USPIO incubation. The image clearly shows a red nucleus and a blue cytoplasmic inclusion with typical perinuclear localization that indicate the presence of USPIO within the cells (magnification $\times 20$, scale bar $50 \mu \mathrm{m}$ ). (B) Percentage of labeled cells after 24 hours and 72 hours of incubation with USPIO. Data expressed as mean \pm SD. (C and D) TEM images of ASC incubated for $72 \mathrm{~h}$ with $200 \mu \mathrm{gg}$ Fe/ml of USPIO. In C, the scale bar is $5,000 \mathrm{~nm}$; in $\mathbf{D}$ note the endocytic invagination containing nanoparticles and the internalized nanoparticles inside the endosome (scale bar 2,000 nm). In $\mathbf{C}$ and $\mathbf{D}$ arrows indicate the presence of nanoparticles inside the cell. (E) TEM image of unlabeled ASCs as negative control (scale bar 5,000 nm).

Abbreviations: ASC, adipose stem cell; TEM, transmission electron microscopy; USPIO, ultrasmall superparamagnetic iron oxide nanoparticles. 
At 72 hours of incubation, intracellular iron content in ASC-USPIO was $10.52 \pm 0.66 \mathrm{pg} / \mathrm{cell}, 11.22 \pm 0.013 \mathrm{pg} / \mathrm{cell}$, and $14.77 \pm 0.021 \mathrm{pg} / \mathrm{cell}$ (data expressed as mean $\pm \mathrm{SD}$ ) at concentrations of $50 \mu \mathrm{g} \mathrm{Fe} / \mathrm{mL}, 100 \mu \mathrm{g} \mathrm{Fe} / \mathrm{mL}$, and $200 \mu \mathrm{g} \mathrm{Fe} / \mathrm{mL}$, respectively. These data are consistent with the total iron content reported in the literature for iron oxidelabeled cells. ${ }^{26}$ Moreover, the results obtained from the quantification of intracellular iron were in line with PB staining: a dose- and time-dependent uptake of USPIO was detected.

\section{MRI of ASC-USPIO}

$\mathrm{T}_{2}$ parametric maps were acquired in gel phantoms containing ASC-USPIO in order to determine the optimal experimental parameters for MRI detection, in terms of both incubation time and iron concentration. The analysis of $\mathrm{R} 2$ values $(1 / \mathrm{s})$ revealed an incubation time and USPIO concentration-dependent behavior (Figure 2A). After 24 hours of incubation, a significant difference $(P<0.05)$ in $\mathrm{R} 2$ values was detected between $100 \mu \mathrm{g} \mathrm{Fe} / \mathrm{mL}$ and $200 \mu \mathrm{g} \mathrm{Fe} / \mathrm{mL}$. After 72 hours of incubation, ASC-USPIO phantoms exhibited significant $(P<0.05)$ differences in R2 values between phantoms containing cells labeled with $50 \mu \mathrm{g} \mathrm{Fe} / \mathrm{mL}, 100 \mu \mathrm{g} \mathrm{Fe} / \mathrm{mL}$, or $200 \mu \mathrm{g} \mathrm{Fe} / \mathrm{mL}$. Moreover, no significant difference in $\mathrm{R} 2$ values was detected between 24 hours and 72 hours of incubation at $200 \mu \mathrm{g} \mathrm{Fe} / \mathrm{mL}$. MRI signal of unlabeled cells was comparable to water signal.

A

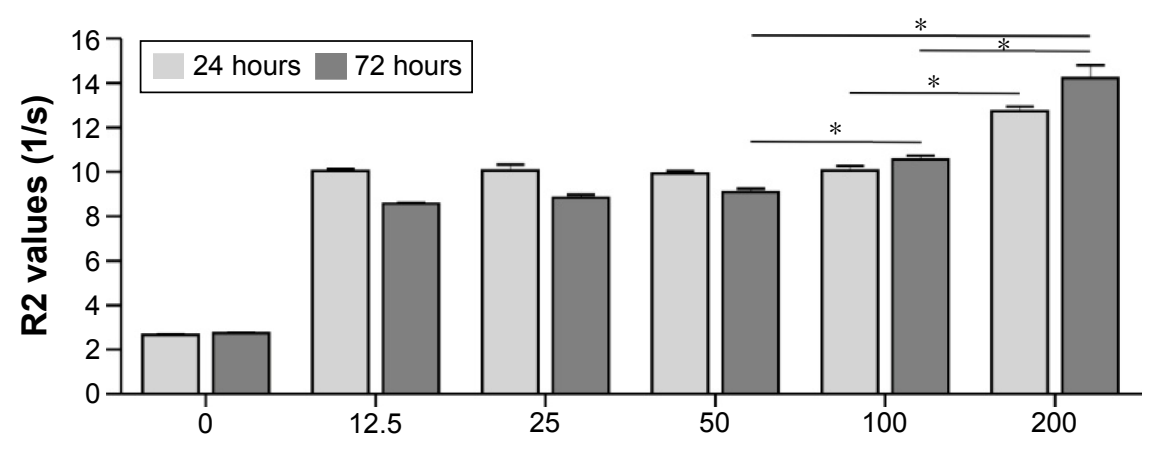

B

USPIO concentrations ( $\mu \mathrm{g} \mathrm{Fe} / \mathrm{mL}$ )

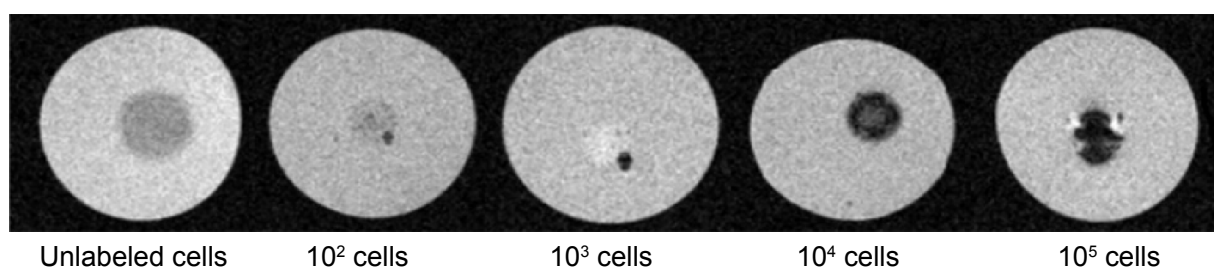

C
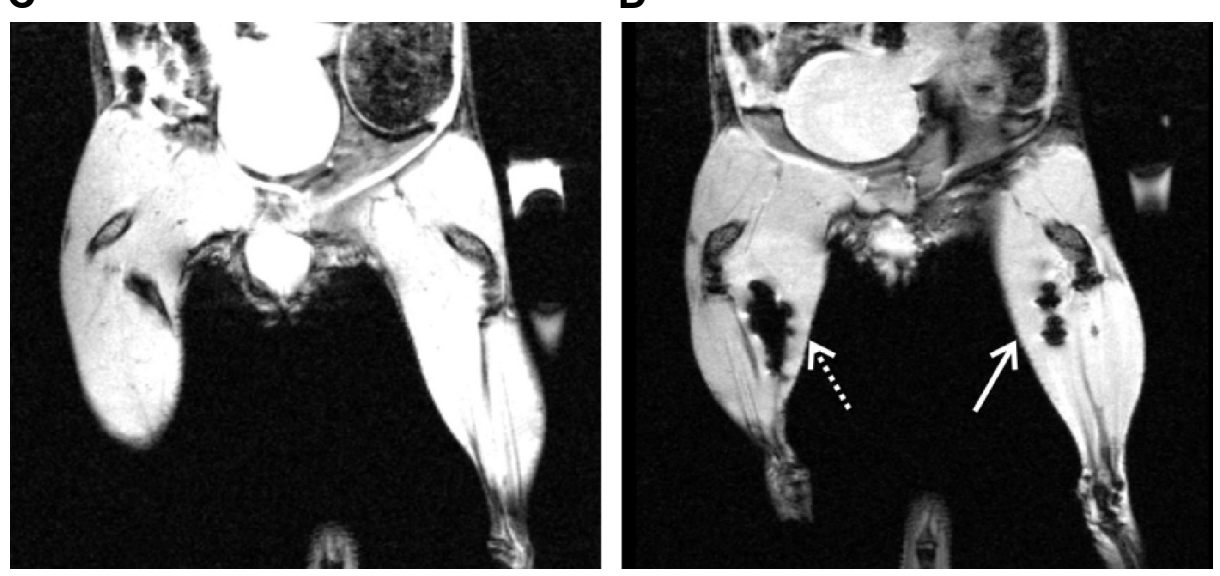

Figure 2 MRI of ASC-USPIO.

Notes: (A) R2 values (I/s) of ASCs unlabeled and labeled with increasing USPIO concentrations ( $12.5 \mu \mathrm{g} \mathrm{Fe} / \mathrm{mL}, 25 \mu \mathrm{g} \mathrm{Fe} / \mathrm{mL}, 50 \mu \mathrm{g} \mathrm{Fe} / \mathrm{mL}, 100 \mu \mathrm{g} \mathrm{Fe} / \mathrm{mL}$, and $200 \mu \mathrm{g} \mathrm{Fe} / \mathrm{mL}$ ) after 24 hours and 72 hours of incubation. Data expressed as mean \pm SD. $* P<0.05$. R2, relaxivity (I/s) of ASC-USPIO nanoparticles. (B) Representative MR images of gel phantom containing labeled ASCs acquired for the evaluation of the detection limit. Different amounts (from $10^{2}$ to $10^{5}$ cells) of ASC-USPIO were used. MRI can efficiently detect up to $10^{2}$ cells in vitro. In vivo MR images of acquired preintramuscular (C) and postintramuscular (D) injections of $5 \times 10^{3}$ (dotted arrow) and $2.5 \times 10^{3}$ (arrow) ASC-USPIO. After intramuscular injection (D), we detected up to $2.5 \times 10^{3}$ ASC-USPIO.

Abbreviations: ASC, adipose stem cell; MR, magnetic resonance; MRI, magnetic resonance imaging; USPIO, ultrasmall superparamagnetic iron oxide nanoparticles. 
Based on the results described earlier and the lack of significant effects on cell viability, a combination of $200 \mu \mathrm{g}$ $\mathrm{Fe} / \mathrm{mL}$ of USPIO and 72 hours of incubation time was chosen for further experiments.

In order to test the detectability and evaluate the detection limit of ASC-USPIO with MRI, decreasing numbers of labeled ASCs $\left(10^{5}, 10^{4}, 10^{3}\right.$, and $10^{2}$ cells) were immobilized in different gel tubes (agarose at 2\% w/w, $1.5 \mathrm{~mL}$ ). Tubes containing unlabeled cells and free agarose gel were used as control. $\mathrm{T}_{2}{ }^{*}$-weighted images of the tubes containing ASCUSPIO revealed the presence of hypointense spots, attributable to the presence of iron nanoparticles. These results showed that, by using the labeling protocol described earlier, MRI can efficiently detect a small amount of ASC-USPIO, down to $10^{2}$ cells in vitro (Figure 2B). Unlabeled cells were not detectable in MR images.

Having established a protocol allowing the efficient detection of ASCs in vitro, we carried out further studies aimed at understanding whether USPIO-labeled cells could be localized by MRI in muscle tissues. For in vivo experiments, two different amounts of USPIO-labeled or unlabeled ASCs $\left(2.5 \times 10^{3}\right.$ and $\left.5 \times 10^{3}\right)$ were intramuscularly injected in the hind limbs of mice. By using this method, we were able to detect $2.5 \times 10^{3}$ ASC-USPIO (Figure $2 \mathrm{C}$ and D).

\section{Isolation and characterization of exosomes-USPIO}

Exosomes were isolated from labeled ASC supernatants obtained after USPIO incubation of 24 hours. In particular, whereas R2 values of ASC-USPIO after 24 hours and 72 hours of incubation were not statistically significantly different and, above all, to avoid a long incubation time (72 hours of nanoparticles incubation +48 hours of FBS deprivation) that could induce cell suffering, we decided to perform a USPIO incubation of 24 hours. Exosomes were quantified using protein concentration: for each isolation, the yield was $100-150 \mu \mathrm{g} / \mathrm{mL}$ of exosomal proteins. Western blot analysis of isolated vesicles showed a signal at $70 \mathrm{kDa}$ and $95 \mathrm{kDa}$, after incubation with HSP70 and Alix antibodies, respectively (Figure 3A), confirming that isolated vesicles were exosomes. We then performed ultrastructural morphology analysis by TEM, in order to prove that the exosomal fraction still contained USPIO. TEM images clearly showed dark spots inside the vesicles, confirming the presence of iron nanoparticles (labeled exosomes) (Figure 3C). Finally, using the spectrophotometric method, exosomes-USPIO were found to contain $0.643 \mu \mathrm{g}$ of iron per $100 \mu \mathrm{g}$ of exosomal proteins.

\section{MRI of exosomes-USPIO}

After isolation and characterization, $3 \mu \mathrm{g}$ of exosomesUSPIO were immobilized in a gel matrix (agarose at $2 \%$ $\mathrm{w} / \mathrm{w}) . \mathrm{T}_{2}{ }^{*}$-weighted images were used to assess the presence of exosomes-USPIO with MRI. Exosomes were detectable as a hypointense spot in the gel matrix (Figure 3B). Unlabeled exosomes immobilized in an agarose gel were used as control. In order to confirm that MR images really reported exosomes-USPIO, we performed TEM on the portion of gel visualized as a hypointense spot in MRI. TEM images clearly confirmed the presence of labeled exosomes within the agarose gel (Figure 3C).

Having efficiently visualized exosomes-USPIO in vitro, we focused on the in vivo MRI detection of labeled exosomes
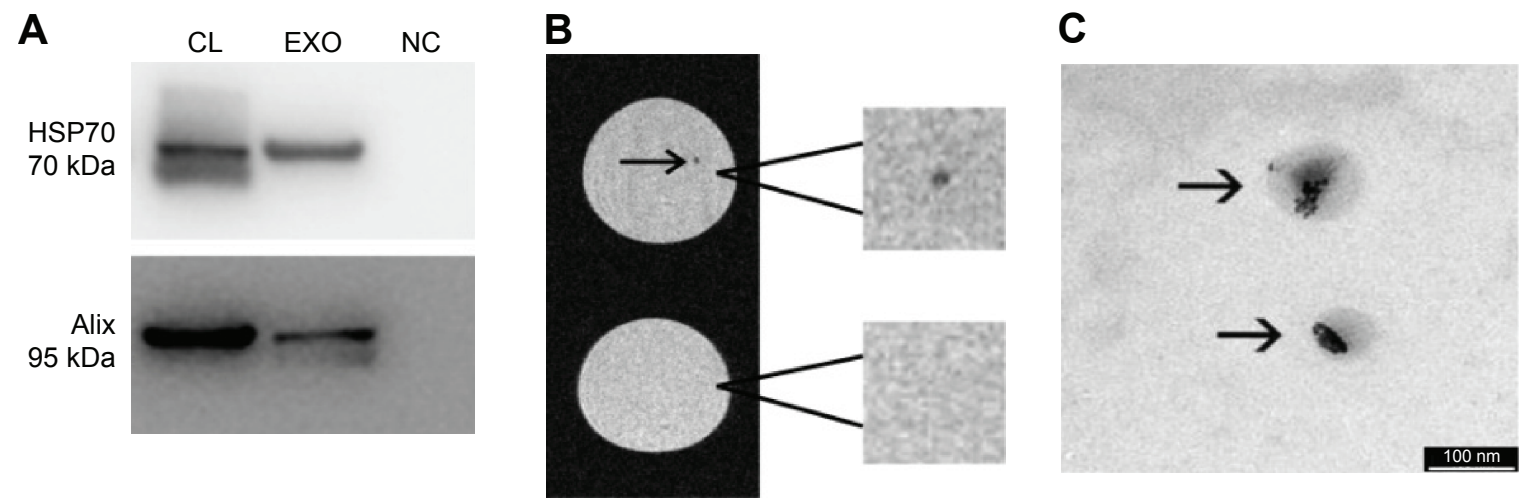

Figure 3 Characterization and visualization of exosomes-USPIO.

Notes: (A) Western blot analysis of the expression of HSP70 (70 kDa) and Alix (95 kDa) in exosomes. Supernatant lacking the ASC and exosomes was used as NC. (B) On the left, MR images of exosomes-USPIO (top) and unlabeled exosomes (bottom) immobilized in gel are shown. The square images on the right show a higher magnification of the areas of interest in each MR image. (C) TEM image of exosomes (arrows) previously visualized by MRI in gel (B) and finally processed for electron microscopy; they are still labeled with nanoparticles (scale bar $100 \mathrm{~nm}$ ).

Abbreviations: ASC, adipose stem cell; CL, ASC cell lysate; EXO, exosomes; MR, magnetic resonance; MRI, magnetic resonance imaging; NC, negative control; TEM, transmission electron microscopy; USPIO, ultrasmall superparamagnetic iron oxide nanoparticles. 

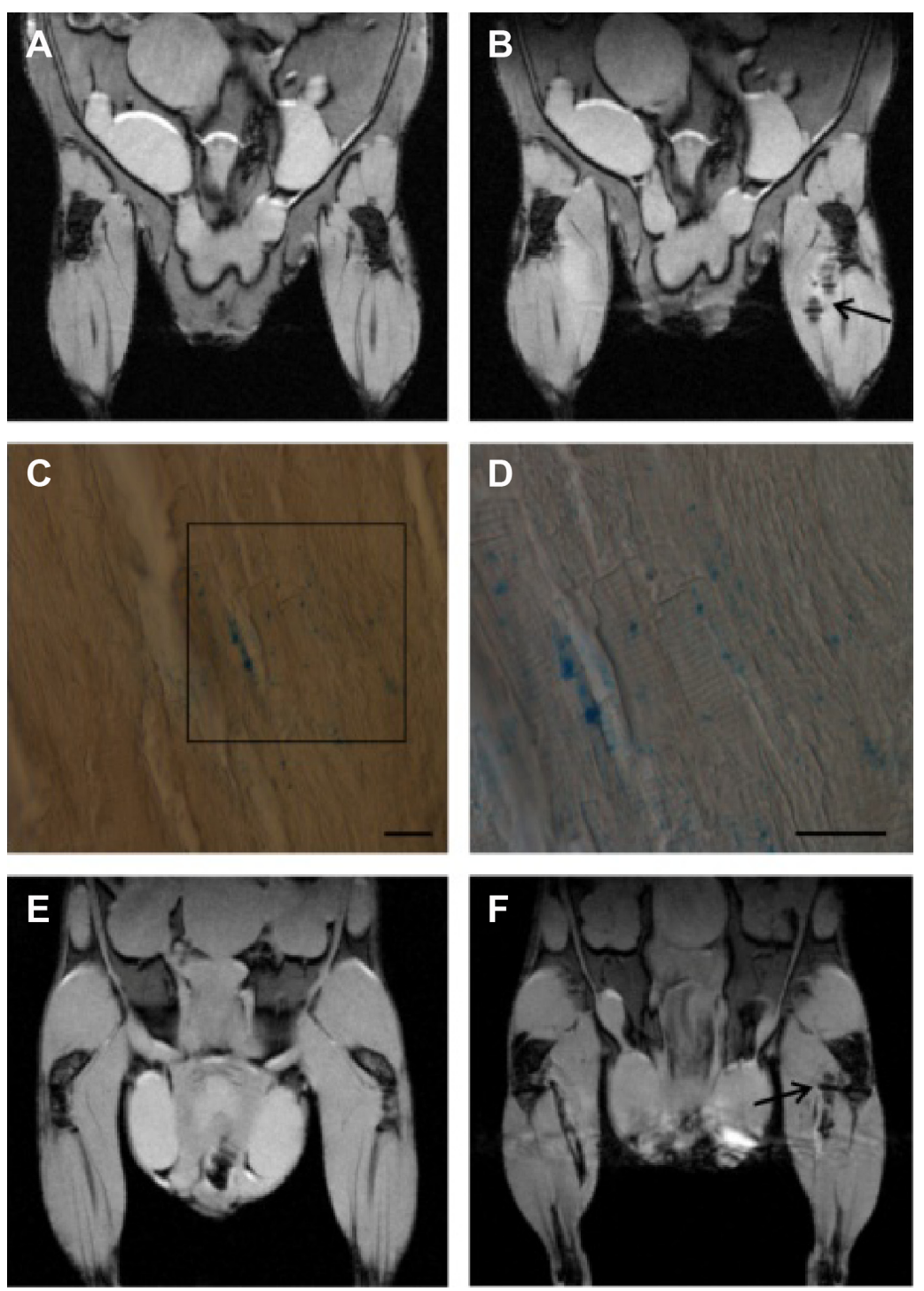

Figure 4 In vivo MRI of exosomes-USPIO.

Notes: In vivo MR images acquired preintramuscular (A) and postintramuscular (B) injections of exosomes-USPIO (arrow). Prussian blue histological examination of extracted muscle tissue: blue spots inside the muscle confirmed the presence of iron nanoparticles (C) (magnification $\times 20$, scale bar $50 \mu \mathrm{m})$. $\mathbf{D}$ shows a higher magnification $(\times 40$, scale bar $50 \mu \mathrm{m})$ of the boxed area shown in $\mathbf{C}$. In vivo MR images acquired preintramuscular (E) and postintramuscular (F) injections of plain USPIO (arrow) containing the same amount of iron of labeled exosomes; the signal is comparable with that detected in exosomes-USPIO (B).

Abbreviations: MR, magnetic resonance; MRI, magnetic resonance imaging; USPIO, ultrasmall superparamagnetic iron oxide nanoparticles.

in mice after intramuscular injection of labeled exosomes. Coronal $\mathrm{T}_{2} *$-weighted images of hind limbs pre- and postintramuscular injections of $5 \mu \mathrm{g}$ of exosomes-USPIO are shown in Figure 4A and B. In vivo MR images revealed that exosomes-USPIO were clearly detectable in the muscular tissue. Histological examination of gastrocnemius confirmed the presence of iron: PB staining showed several blue spots inside the muscle fibers in a spatially limited region close to the injection site (Figure $4 \mathrm{C}$ and D). In mice receiving an intramuscular injection of plain USPIO containing the same amount of iron of labeled exosomes, the MR images clearly showed localized loss of signal comparable with that detected in images of mice injected with exosomes-USPIO, confirming the high sensitivity of MRI (Figure 4E and F).

\section{Discussion}

The safe and easy availability of ASCs and their ability to migrate to damaged tissues and contribute to reparative processes render these cells of great interest in neurodegenerative diseases. In the last decade, several studies have demonstrated that the therapeutic potential of ASCs could be due to their paracrine activity rather than to their integration and survival in host tissues. ${ }^{46}$ In particular, the paracrine mechanism seems to be mediated through exosomes release, ${ }^{3}$ 
making exosomes a potential alternative to cell therapy in neurodegenerative diseases.

The ability to visualize and track exosomes with a noninvasive tool, such as MRI, ${ }^{27}$ would be key for the understanding of the biological mechanisms (eg, homing, functional capabilities, and site of action), which are at the basis of their potential. To this purpose, we have set up a new method to label exosomes with iron oxide nanoparticles. USPIO are an optimal candidate for exosomes labeling: they are stable, biocompatible, ranging in size from $5 \mathrm{~nm}$ to $7 \mathrm{~nm}$, and hence sufficiently small to be incorporated in exosomes. ${ }^{27}$ Recently, USPIO have been used to label exosomes by the electroporation technique, ${ }^{.3}$ to date, despite variable results, ${ }^{25}$ electroporation is one of the most frequently used methods to load cells and exosomes with cargo. ${ }^{23,28}$ However, electroporation may affect membrane integrity since the membrane is exposed to a strong electric field to cause spontaneous pore formation. It is well established that exosomes carry out their biological function by transferring their content to the target cell. This mechanism requires first, interaction between exosomes and cell membranes and second, membranes' fusion and exosomes' internalization. ${ }^{10,11}$ For this reason, it is fundamental to preserve the integrity of the exosome membrane. In our study, we propose a new protocol to label exosomes with USPIO starting by labeling cells, which avoids direct manipulation of exosome membranes.

First of all, we optimized the parameters to label ASCs in terms of cell viability, labeling efficiency, iron content, and MR image contrast. TEM images showed that labeled ASCs incorporate USPIO by endocytic mechanisms, as already reported..$^{29}$ It has been demonstrated that nanoparticles internalized by endocytosis often accumulate inside multivesicular bodies, ${ }^{30}$ which, in turn, may fuse with the plasma membrane thus releasing their cargo (exosomes). ${ }^{31}$ It is likely that USPIO undergo the same secretion process of the endosomal system.

MRI experiments were designed to find the highest detectable nanoparticle concentrations compatible with cell viability and to establish the lowest detectable USPIO-labeled cell numbers. Our results showed that MRI is able to visualize $10^{2}$-labeled cells, an amount that is several orders of magnitude lower than what is normally used in in vivo animal disease models..$^{4-6}$ The next step was to evaluate whether exosomes isolated from ASC-USPIO retain nanoparticles and if they could be visualized by MRI. In vitro MR images of exosomes-USPIO showed a localized hypointense region, attributable to the presence of iron nanoparticles, as confirmed by TEM. Moreover, we were able to detect exosomes-USPIO in vivo with MRI after intramuscular injection. The magnetic susceptibility artifact had the typical shape of the magnetic dipole field lines. A similar susceptibility artifact appeared when an equivalent amount of USPIO (in terms of iron content) was injected. The presence of USPIO was confirmed by the histological analysis of muscular tissues.

\section{Conclusion}

The production of labeled exosomes through labeled cells does not alter the vesicles membrane and therefore allows preservation of their integrity. This innovative method opens up the possibility of tracking exosomes in vivo with a powerful and noninvasive technique, such as MRI, and could represent a significant step forward to the unveiling of exosome homing and, more broadly, their role in normal and pathological conditions.

\section{Acknowledgments}

This work was supported by Fondazione Cariverona (Verona, Italy) through "Verona Nanomedicine Initiative" Project and by MIUR through FIRB Project RBAP114AMK - RI.NA. ME. "Rete Integrata per la Nanomedicina". The authors would like to thank Doctor Federico Boschi for his support and advice in the statistical analysis.

\section{Disclosure}

The authors report no conflicts of interest in this work.

\section{References}

1. Tsuji W, Rubin JP, Marra KJ. Adipose-derived stem cells: implications in tissue regeneration. World J Stem Cells. 2014;6(3):312-321.

2. Peroni D, Scambi I, Pasini A, et al. Stem molecular signature of adiposederived stromal cells. Exp Cell Res. 2008;314(3):603-615.

3. Baglio SR, Pegtel DM, Baldini N. Mesenchymal stem cell secreted vesicles provide novel opportunities in (stem) cell-free therapy. Front Physiol. 2012;3:359-371.

4. Constantin G, Marconi S, Rossi B, et al. Adipose-derived mesenchymal stem cells ameliorate chronic experimental autoimmune encephalomyelitis. Stem Cell. 2009;27(10):2624-2635.

5. Marconi S, Castiglione G, Turano E, et al. Human adipose-derived mesenchymal stem cells systemically injected promote peripheral nerve regeneration in the mouse model of sciatic crush. Tissue Eng Part A. 2012;18(11-12):1264-1272.

6. Marconi S, Bonaconsa M, Scambi I, et al. Systemic treatment with adipose-derived mesenchymal stem cells ameliorates clinical and pathological features in the amyotrophic lateral sclerosis murine model. Neuroscience. 2013;248:333-343.

7. Wei X, Zhao L, Zhong J, et al. Adipose stromal cells-secreted neuroprotective media against neuronal apoptosis. Neurosci Lett. 2009; 462(1):76-79

8. Maumus M, Jorgensen C, Noël D. Mesenchymal stem cells in regenerative medicine applied to rheumatic disease: role of secretome and exosomes. Biochimie. 2013;95(12):2229-2234.

9. Lavoie JR, Rosu-Myles M. Uncovering the secrets of mesenchymal stem cells. Biochimie. 2013;95(12):2212-2221. 
10. Kourembanas S. Exosomes: vehicles of intercellular signaling, biomarkers, and vectors of cell therapy. Annu Rev Physiol. 2015;77:13-27.

11. Raposo G, Stoorvogel W. Extracellular vesicles: exosome, microvesicles, and friends. J Cell Biol. 2013;200(4):373-383.

12. Vlassov AV, Magdaleno S, Setterquist R, Conrad R. Exosomes: current knowledge of their composition, biological functions, and diagnostic and therapeutic potentials. Biochim Biophys Acta. 2012;1820(7):940-948.

13. Valadi H, Ekström K, Bossios A, Sjöstrand M, Lee JJ, Lötvall JO. Exosomemediated transfer of mRNAs and microRNAs is a novel mechanism of genetic exchange between cells. Nat Cell Biol. 2007;9(6):654-659.

14. Mathivanan S, Ji H, Simpson RJ. Exosomes: extracellular organelles important in intercellular communication. J Proteomics. 2010; 73(10):1907-1920.

15. Xin H, Li Y, Cui Y, Yang JJ, Zhang ZG, Chopp M. Systemic administration of exosomes released from mesenchymal stromal cells promote functional recovery and neurovascular plasticity after stroke in rats. J Cereb Blood Flow Metab. 2011;31(11):2181-2188.

16. Xin H, Li Y, Buller B, et al. Exosome-mediated transfer of miR-133b from multipotent mesenchymal stromal cells to neural cells contributes to neurite outgrowth. Stem Cells. 2012;30(7):1556-1564.

17. Pusic AD, Kraig RP. Youth and environment enrichmen generate serum exosomes containing miR-219 that promote CNS myelination. Glia. 2014;62(2):284-299.

18. Nguyen PK, Riegler J, Wu JC. Stem cell imaging: from bench to bedside. Cell Stem Cell. 2014;14(4):431-444.

19. Marzola P, Longoni B, Szilagyi E, et al. In vivo visualization of transplanted pancreatic islets by MRI: comparison between in vivo, histological and electron microscopy findings. Contrast Media Mol Imaging. 2009; 4(3):135-142.

20. Neri M, Maderna C, Cavazzin C, et al. Efficient in vitro labeling of human neural precursor cells with superparamagnetic iron oxide particles: relevance for in vivo cell tracking. Stem Cells. 2008;26(2):505-516.
21. Zhang $Z$, Jiang $Q$, Jiang $F$, et al. In vivo magnetic resonance imaging tracks adult neural progenitor cell targeting of brain tumor. Neuroimage. 2004;23(1):281-287.

22. Zhao X, Zhao H, Chen Z, Lan M. Ultrasmall superparamagnetic iron oxide nanoparticles for magnetic resonance imaging contrast agent. J Nanosci Nanotechnol. 2014;14(1):210-220.

23. Hu L, Wickline SA, Hood JL. Magnetic resonance imaging of melanoma exosomes in lymph nodes. Magn Reson Med. 2015;74:266-271.

24. Peng XH, Quian X, Mao H, et al. Targeted magnetic iron oxide nanoparticles for tumor imaging and therapy. Int J Nanomedicine. 2008; 3(3):311-321.

25. Hood JL, Scott MJ, Wickline SA. Maximizing exosomes colloidal stability following electroporation. Anal Biochem. 2014;448:41-49.

26. Rad AM, Arab AS, Iskander ASM, Jiang Q, Soltanian-Zadeh H. Quantification of superparamagnetic iron oxide (SPIO)-labeled cells using MRI. J Magn Reson Imaging. 2007;26(2):366-374.

27. Bull E, Madani SY, Sheth R, Seifalian A, Green M, Seifalian AM. Stem cell tracking using iron oxide nanoparticles. Int J Nanomedicine. 2014;9:1641-1653.

28. Tian Y, Li S, Song J, et al. A doxorubicin delivering platform using ingeneered natural membrane vesicle exosomes for targeted tumor therapy. Biomaterials. 2014;35(7):2383-2390.

29. Alam SR, Stirrat C, Richards J, et al. Vascular and plaque imaging with ultrasmall superparamagnetic particles of iron oxide. J Cardiovasc Magn Reson. 2015;17:83-92.

30. Malatesta M, Giagnacovo M, Costanzo M, et al. Diaminobenzidine photoconversion is a suitable tool for tracking the intracellular location of fluorescently labelled nanoparticles at transmission electron microscopy. Eur J Histochem. 2012;56(2):e20.

31. Keller S, Sanderson MP, Stoeck A, Altevogt P. Exosomes: from biogenesis and secretion to biological function. Immunol Lett. 2006;107(2): 102-108.
International Journal of Nanomedicine

\section{Publish your work in this journal}

The International Journal of Nanomedicine is an international, peerreviewed journal focusing on the application of nanotechnology in diagnostics, therapeutics, and drug delivery systems throughout the biomedical field. This journal is indexed on PubMed Central, MedLine, CAS, SciSearch ${ }^{\circledR}$, Current Contents ${ }^{\circledR} /$ Clinical Medicine,

\section{Dovepress}

Journal Citation Reports/Science Edition, EMBase, Scopus and the Elsevier Bibliographic databases. The manuscript management system is completely online and includes a very quick and fair peer-review system, which is all easy to use. Visit http://www.dovepress.com/ testimonials.php to read real quotes from published authors. 